\title{
LA OBRA EL MANIFIESTO CONTRASEXUAL EN EL IDEAL DEL FEMINISMO MODERNO
}

\author{
THE CONTRASEXUAL MANIFESTO IN THE IDEAL OF MODERN FEMINISM
}

\author{
Douglas Verbicaro Soares \\ Universidade Federal de Roraima, Boa Vista, RR, Brasil, douglas_verbicaro@yahoo.com.br
}

Ana Beatriz Freitas Silva

Universidade Federal do Pará, Belém, PA, Brasil, abe.freitas04@gmail.com

María Ángeles Herrero Uña

Universidad de Salamanca, Cyl, Espanha, elmoguete@gmail.com

DOI: http://dx.doi.org/10.31512/missioneira.v22i1.220

Recebido em: 13/012/2019

Aceito em: 14/06/2020

\begin{abstract}
Resumen: La obra el Manifiesto Contrasexual de Paul B. Preciado promueve, en la sociedad internacional, un cuestionamiento en cuanto a la práctica sexual basada en valores heteronormativos conservadores. Condiciones éstas que inhiben la libertad sexual de los cuerpos. Por esta razón, son novedosos los estudios que promuevan cambios importantes, principalmente, en el modo de entender el sexo y la sexualidad, relacionándolo con el placer sexual, rompiendo con ciertos valores tradicionales históricos. Valores que justificaron la opresión sexual, las diferencias comportamentales de género y, sobre todo, la exclusión participativa de las minorías sexuales por orientación sexual e identidad de género. En este íntimo, se utilizó la metodología de la revisión bibliográfica, buscando comprender las contribuciones del trabajo de Preciado, para la comprensión del género y de la sexualidad.
\end{abstract}

Palavras clave: Sexo. Sexualidad. Género. Discriminación.

\begin{abstract}
The work of Paul B. Preciado Contra-Sexual Manifesto promotes, in international society, a questioning how much sexual practice based on conservative heteronormative values. Conditions those that inhibit the sexual freedom of the bodies. For this reason, the new studies are important to make changes, mainly, in the way of understanding the sex, relating it to sexual pleasure, breaking with traditional historical values. Values that justified the own sexual oppression, behavioral gender differences and, above all, participatory exclusion of sexual minorities on sexual orientation and gender identity.
\end{abstract}

Keywords: Sex. Sexuality. Gender. Discrimination. 


\section{Introducción}

Objetivo de este trabajo es visibilizar la obra el Manifiesto Contrasexual, de Paul. B. Preciado, como un instrumento didáctico que permite una reformulación y concientización social sobre temas de sexo, sexualidad humana, educación para la igualdad de género y Derechos Humanos. ${ }^{1}$

Es imperioso argumentar que la obra trabajada podrá ser empleada como alternativa para el cuestionamiento socio-cultural e histórico sobre sexo, género, en especial sobre libertad sexual y orientaciones sexuales. Así, su relevancia estaría en el hecho de representar un temario bastante discutido en sociedades por el mundo, que engloba a existencia de numerosos factores que dificultan la protección y real aplicación de derechos para un número considerable de personas. Ciudadanos estos victimados todos los días, por cuestiones que tratan sobre el sexo y violaciones a principios democráticos de igualdad, libertad, privacidad, solidaridad y dignidad humana.

El libro Manifiesto Contrasexual de Preciado impulsó el desarrollo de una nueva interpretación sobre género y libertad sexual, sobre todo para las mujeres. La vivencia de la sexualidad para las mujeres no ha sido sencilla, ya que, a lo largo de la propia historia de humanidad, las mujeres han sido consideradas como ciudadanas de segunda categoría en relación con los varones. Las mujeres han sido impedidas de alcanzar los mismos derechos que a los varones, donde el valor del sexo masculino y el rol por él ejercido, en diversas sociedades, valían más que la igualdad entre los/as ciudadanos/as.

Es importante enfatizar que los trabajos de investigación creados para desmitificar las cuestiones de género, así como los estudios sobre las mujeres, han permitido la ampliación de la visibilidad de cuestiones relevantes socialmente, muchas de ellas de origen en los movimientos feministas que reivindicaban la efectiva igualdad entre las personas (MAQUIEIRA, 2005, p. 284).

Los desafíos a ser enfrentados son todavía costosos, pero dignos de ser realizados, una vez que son indispensables para las sociedades más justas, libres y harmónicas.

Es importante reconocer que, en las luchas de movimientos de grupos organizados contemporáneos, entre ellos: el de feministas, feministas lesbianas o de grupos homosexuales, vienen apuntando por la búsqueda de alternativas para frenar las desigualdades entre los sexos y, así, propiciar el pleno desarrollo de la libertad sexual de las personas.

1 La persona que recibió el nombre Beatriz Preciado en España, en los años 70, anunció en 2014, que estaba realizando un proceso de transición de identidad. Así, implementando el cambio de su primer nombre de Beatriz para Paul, tornándose Paul B. Preciado. 
El objetivo del presente artículo es de visibilizar novedosas iniciativas para la concientización y sensibilización sobre igualdad de género y libertad sexual han sido incrementados en las últimas décadas, lo que posibilitó mejorías en las situaciones de exclusión participativa de las mujeres y colectivos víctimas de discriminación, como por ejemplo las mujeres lesbianas, que sufren una doble problemática, por ser mujeres y por tener una orientación diversa de la heterosexual dominante en el mundo moderno.

Por esa razón, algunos autores, como Segarra y Carabí, aducen que:

Los estudios sobre las relaciones de género contribuyen para comprenderse las dificultades que sufren las mujeres en sociedad y, también, el difícil trabajo de los movimientos con ideales de igualdad entre los géneros (SEGARRA; CARABÍ, 2000, p. 43).

En ese sentido, este trabajo enfocará la educación como instrumento de emancipación ciudadana para concientizar las diversas sociedades sobre los temas de género y orientación sexual, dando especial atención al combate a los obstáculos socio-culturales existentes que impiden la participación igualitaria entre los individuos en diferentes partes del mundo. Para ello, se adoptaron los procedimientos metodológicos de la investigación documental y bibliográfica (LAKATOS, MARCONI, 2010), a través de los métodos deductivo y comparativo (GIL, 2008), eligiendo en ese contexto, obras que se presentaran como marcos para el feminismo moderno, y relacionando a las obras de Preciado, para resaltar, su importancia y contribuciones.

El artículo comienza con breve presentación del autor, las temáticas abordadas y su importancia, pasando por el enfoque directo en la obra aquí analizada, el Manifiesto Contrassexual. En consecuencia, se explicitan motivos, razones por las cuales esta obra presenta relevancia, cómo puede contribuir a la comprensión diferenciada del género y de las sexualidades, para posteriormente explicitar la relación de la temática que en el contexto teórico del manifiesto, como perspectiva de cambio en la comprensión de la sexualidad. Por último, se relaciona el trabajo de Preciado, a los presupuestos del feminismo moderno, a las relaciones de la sexualidad humana ya la necesidad de ruptura del paradigma sexual, con vistas a las conclusiones finales del trabajo.

\section{Los estudios de Preciado}

Se hacen importantes algunas consideraciones cuanto a Preciado en ese apartado. El autor español ha desarrollado diversos trabajos en la esfera de la sexualidad humana y género. Podemos incluso citar algunas de sus obras más destacables, como ha sido: Pornotopía, Testo Yonqui y Manifiesto Contrasexual. La relevancia de sus estudios ha permitido una reflexión universal cuanto a la temática del sexo, los feminismos y los Derechos Humanos. 
Es necesario pensar el sexo, al menos a partir del siglo XVIII, como una tecnología biopolítica. Es decir, como un sistema complejo de estructuras reguladoras que controlan la relación entre los cuerpos, los instrumentos, las máquinas, los usos y los usuarios (PRECIADO, 2011, p. 68).

Concordamos con que la contextualización socio-política, cultural y religiosa ha propiciado la formación de la temática sexual, dividiendo los roles del masculino y femenino, atribuyendo al segundo una connotación de desprestigio, como se fuera de segunda categoría, siendo, por lo tanto, inferior a lo masculino. Ese hecho histórico discriminador ha permitido la exclusión participativa de las mujeres y en el trato desigual entre los géneros.

Por esa razón, surgieron a lo largo de los siglos, reivindicaciones para romper con el modelo jerárquico desigual establecido por normas patriarcales, donde el género y sus estudios, pasan a nortear los clamores históricos para la construcción de modelos sociales para la integración en igualdad de las mujeres en sociedad.

No se puedo olvidar que inicialmente para los temas para lograr a igualdad de género se buscó la equiparación de las mujeres a los mismos derechos que los varones. Hoy, las búsquedas están en visibilizar las desigualdades materiales existentes que obstaculizan la efectiva igualdad entre mujeres y varones. De esa forma, los movimientos feministas propiciaron cambios en las estructuras sociales, en el sentido de lograr cierta, pero limitada igualdad de trato entre varones y mujeres, en distintos temas relacionados con la el género y sexualidad humana. No obstante, reconocemos que mucho todavía hay se hacerse para tener una verdadera igualdad.

\section{Conociendo la obra de Preciado}

Preciado critica, principalmente, la fuerte influencia del modelo heterosexual, comprendido como dominante frente a los demás existentes, como pueden ser la bisexualidad u homosexualidad, que históricamente, han ocupado posiciones de sumisión a los ideales heteronormativos.

En su obra, Preciado propone una nueva forma de exteriorizar la sexualidad humana, así, podemos hablar una nueva interpretación sobre masculinidades y feminidades, relacionada con la sexualidad humana. En este sentido, el autor nos enseña que no debemos solamente mirar la sexualidad en valores físicos, visuales, estéticos del sexo, sino abrir la comprensión sobra la exteriorización sexual de las personas y la búsqueda por alternativas en se descubrir el placer sexual.

De acuerdo con esa visión, habla de la existencia de la multiplicidad de los cuerpos y sus distintas formas de manifestación, ya no solamente al masculino y al femenino, una vez que la categoría de género ha conducido a la restricción de los conceptos multíplices trabajados. 
Siendo, por lo tanto, un mecanismo que amplía a las cuestiones que versan sobre sexualidad humana, roles, identidades de género y diversidad de orientación sexual (YOUTUBE, 2006).

Con base en estas ideas, el propio Paul B. Preciado define su trabajo como una retraducción política de determinados trabajos científicos: una de las cuestiones políticas más urgentes hoy es precisamente la que suscita la relación entre las políticas de identidad y las estrategias queer.

La comunidad LGBT, junto con los temas de identidad de género y estudios de género, tienen una proyección discursiva en la actualidad, haciendo con que la obra Manifiesto Contrasexual se coloque dentro de éste giro de ideas, haciendo con que nuevas discusiones salgan adelante en los más variados sectores, sean ellos en los políticos, económicos, sociales, jurídicos, educativos, etcétera. Por así decir, ocurre un giro con relación al régimen de ocultamiento y secreto que se basaban las ideas disciplinares, antiguas del siglo XIX, que se produjeron con las identidades sexuales (homosexual y heterosexual) principalmente.

Verdaderos cambios vienen siendo notados: desde la criminalización social de las identidades de géneros, hasta la búsqueda por un real trato igualitario entre todos los ciudadanos, en pleno siglo XXI.

Un momento de progresiva normalización y asimilación de las minorías sexuales en la norma (matrimonio gay, igualdad legal, representación mediática) parece políticamente incongruente seguir considerando a los gais y las lesbianas como un grupo político radical (CARRILLO, 2010, p. 47-71).

En ese sentido, su visón de interpretación estaría dentro del feminismo moderno, junto con los temas de orientación sexual, en especial la homosexualidad femenina, en una interpretación que está más cerca del ideario queer de Reino Unido o Estados Unidos de América. De este modo, haciendo que grupos sociales tuviesen mejoras en la situación de desprestigio social en la cual viven, la gran mayoría de las mujeres por el mundo, como también, las mujeres lesbianas, caracterizadas históricamente como un colectivo que sigue sufriendo exclusión motivados tanto por ser de mujeres, y además por lesbianas.

Es oportuno alegar que las lesbianas pertenecen a un grupo todavía estigmatizado, que tiene que convivir con obstáculos aún estructurados dentro de valores conservadores, que impiden en trato igualitario entre varones y mujeres. Así como el trato igualitario, también, entre las propias mujeres. En este sentido:

El abordaje que incide sobre las mujeres lesbianas, poniéndolas como marginales, tanto en la geografía feminista, como en las geografías de las sexualidades sigue presente en la actualidad. Por menos desarrollada que sea, representa un campo de exploración científica importante para superar las relaciones de poder e injusticias que marcaron la vida de las mujeres que escapan a la heteronormatividad (SILVA; ORNAT, 2017, p. 424). 
Es posible analizar con estos hechos que ha sido posible la manifestación de otras formas de expresión de este movimiento, lo que rompió con las formas tradicionales y, supuestamente, inmodificables de las tradiciones del movimiento feminista por diferentes sociedades, en especial en Norteamérica.

Los cambios sugeridos pasan desde las aportaciones didácticas dejadas por Judith Butler hasta con los estudios de la actualidad, que han permitido ampliar la visión del género y sexualidad, evidenciando la existencia de un modelo o performance de género, modificado el mundo de lo estético, y otro con una redefinición del cuerpo, de la vida y su propia relación entre sí.

Para Butler, la expresión del género es realizada por conductas que producen resultados. Esas variaciones, en el tiempo y, dentro de una determinada sociedad culturalmente, implican na formación de los roles atribuidos a hombres y mujeres, que definen en muchos de los casos lo que será establecido como típicos del femenino o masculino (BUTLER, 1993). Corroborando con esa idea, Moira Pérez argumenta que:

La reflexión teórica acerca de las diversidades sexogenéricas [...] han recorrido un largo camino en su tarea de detectar, denunciar y desmantelar importantes aspectos de las regulaciones que afectan a los cuerpos, las sexualidades y los géneros [...] y los moldes en que cada una de estas categorías se relaciona con la otra, y los esenciales que les son asignados culturalmente (PÉREZ, 2017, p. 435).

Por esa razón, movimientos organizados, como de las feministas reivindican participación inclusiva (acceso a derechos y a supresión de los obstáculos que generen el trato desigual entre los géneros), cuestionaron el modelo del sexo (patriarcal, conservador y heterosexual con único objetivo reproductivo), como, también, implementaron luchas por la descubierta de la sexualidad (derecho de usar el propio cuerpo por la búsqueda del placer individual), en sociedades por el mundo. Estos cambios han sido notados a lo largo de la historia de la humanidad:

Al analizar la temática de la diversidad en la sexualidad humana, la historia nos ha mostrado que tanto para las mujeres como para los homosexuales (hombres y mujeres), que esas personas han tenido una apertura en los lemas de poder desarrollarse y manifestarse socialmente de un modo más libre. Esa evidencia social, para esa población, ha abierto camino a las reivindicaciones a lo largo de los tiempos por mejores condiciones de vida y de un trato igualitario (VERBICARO SOARES, 2015, p. 49).

De ese modo, la obra de Paul B. viene a aclarar sobre la externalización pública de las transformaciones corporales en los más diferentes ámbitos. Preciado es responsable por enfatizar nuevas definiciones sobre la sexualidad dentro del feminismo moderno:

La definición del biotecnopolitico del cuerpo como una prótesis sexual, una verdadera llamada al ecologismo político, una existencia corporal que ha sido motivada por la 
La Obra el Manifiesto Contrasexual en el Ideal del Feminismo Moderno

Douglas Verbicaro Soares | Ana Beatriz Freitas | Maria de los Ángeles Herrero Uña

constructiva definición del cuerpo como un sistema, de variadas técnicas unidas constituyendo un cuerpo vivo (CARRILLO, 2010, p. 47-71).

La tercera ola del movimiento feminista, a la cual pertenecería la obra de Preciado, engloba las referencias críticas a la heterosexualidad, un modelo que regula la propia sociedad y determina los que serán aceptados y los que no en determinada sociedad, en la cual vinculan sus normatividades heterosexuales frente a las diferentes manifestaciones de la sexualidad humana.

Por esta razón, la obra es importante en la historia de la teoría feminista contrasexual, pues propone un descubrimiento de la sexualidad humana en confronto con los roles previamente establecidos en sociedad, donde la heterosexualidad predomina e impone su fuerza sobre las diversas manifestaciones sexuales humanas, como el más importante y supuestamente natural, basado en ideas patriarcales, machistas, de perjuicios y discriminación.

Es válido recordar que Preciado es conocido por ser una persona que trabaja con el temario queer, relacionada, principalmente, con la conquista de derechos de la comunidad LGBTQI e igualdad de trato para ese colectivo social:

El cuerpo queer pone en cuestión la mitología que garantiza la naturalización de la filiación y la diferencia sexual. En este contexto, el mal sujeto queer genera todo un conjunto de angustias sexuales y políticas. La cuestión de la recepción de las teorías queer en Francia o en España no me preocupa en absoluto porque los límites nacionales no me parecen, en efecto, pertinentes para pensar el trabajo queer (CARRILLO, 2010, p. 47-71).

La obra Manifiesto Contrasexual propone unas prácticas contra-sexuales, o sea, que critican los modelos existentes en sociedad y que sufren una grande imposición del modelo predominante, el heterosexual (normas, costumbres) sobre los demás. En ese sentido, las influencias de Preciado colocarían el sexo en un nivel diferente hasta entonces, ya no sería, solamente, la materialización y opresión de las prácticas con los fines de reproducción humana, pero algo mucho más libertario para el placer humano.

Paul B. Preciado objetiva, con su labor, dar un nuevo enfoque sobre la sexualidad humana, dándole una comprensión más extensa para lograrse la felicidad corporal, sin limitaciones. Por consiguiente, enfatizando que no podemos solamente definir visualmente o físicamente una persona y su sexualidad, sin llevar en consideración la presencia de los cromosomas de las personas, que puedan generar diferentes y múltiples manifestaciones sexuales entre ellas.

Tenemos que llevar en consideración ésta idea y buscar ampliar el concepto de la diversidad sexual, permitiendo la experimentación, principalmente la práctica como esencia de la sexualidad y de las diversas manifestaciones de las identidades de género u orientaciones 
del deseo homosexual. Lo importante en este momento es lograr la aceptación social de esta diversidad y conseguir retirar los obstáculos que impiden la consecución de éste objetivo.

\section{4 ¿El porqué de la viabilidad de si estudiar las referencias del Manifiesto Contrasexual de Preciado? ¿A quién tocará el dildo?}

El trabajo de Preciado es relevante para trazar las primeras ideas de ruptura a los estereotipos creados, a lo largo de los tiempos, cuanto a los temas de la sexualidad humana.

Con la evidencia de las nuevas exigencias democráticas de reconocimiento de las libertades sexuales, la autora rompe con el desconocimiento y alza la idea de la contra sexualidad:

¿Qué es la contra sexualidad?: No es la creación de una nueva naturaleza, sino más bien en fin de la naturaleza como orden que legitima la sujeción de unos cuerpos a otros. La contra sexualidad es, en primer lugar, un análisis crítico de la diferencia de género y de sexo, producto del contrato social heterocentrado, cuyas performatividades normativas han sido inscritas en los cuerpos como verdades biológicas (PRECIADO, 2011, p. 12$3)$.

$\mathrm{Su}$ trabajo innova con el análisis crítico de la diferencia de género y del sexo: demostrando el contrato social basado en características heterosexuales, dando a conocer y a sustituir el contrato social (naturaleza) que conocemos por un contrato contrasexual.

Ya no serían cuerpos de hombres o mujeres y sí cuerpos hablantes. Se hace relevante la citación de las primeras prácticas contra sexuales como posibilidades de una deriva radical con relación al sistema sexo/genero dominante: la utilización de dildos. La erotización del ano y es establecimiento de relaciones $\mathrm{S} \& \mathrm{M}$ contractuales - mutación pos humana del sexo (PRECIADO, 2011, p. 23).

Trabajando con esa interpretación de la sociedad contrasexual, el autor promulga en realidad la desconstrucción de la naturalización de las prácticas sexuales y del sistema de género. Para Preciado:

Esa sociedad proclama la equivalencia, que es diferente de la igualdad de todos los cuerpos-sujetos hablantes que están comprometidos según lo que dice el contrato sexual dedicado al placer-saber, distinto a la de la sexualidad moderna. Sería la manifestación de prácticas alternativas de placer, que no estarían limitadas por las especificaciones de los roles de género, sexo, masculinidades, feminidades. Son un conjunto de mecanismos: máquinas, productos, instrumentos, aparatos, trucos, prótesis, redes, aplicaciones, programas, leyes de circulación etcétera (PRECIADO, 2011, p. 23).

Paul B. Preciado sorprende al enfocar: el principio está en el dildo (sexo plástico), como suplemento que produce aquello que supuestamente debe completar.

Butler (1993) interrelaciona con estas disposiciones, entendiendo de manera similar el sexo como la materialización de una imposición reiterada de normas, lo que abre la suposición de que este proceso será cuestionado y roto cuando: 
Las inestabilidades, las posibilidades de rematerialización, abiertas por este proceso, marcan un área en la cual la fuerza de la ley reguladora puede volverse contra sí misma para generar rearticulaciones que cuestionen la fuerza hegemónica de esa misma ley reguladora (BUTLER, 1993, p. 111).

Es en este mismo contexto que los presupuestos se consolidan en torno a la reformulación de las prácticas sociales, desde el momento en que Preciado (2011) instituyó principios basados en la sociedad contra-sexual, inicialmente basados en el requisito de eliminación de las designaciones masculinas. Son formas femeninas, administrativas y legales de carácter estatal, así como la tarjeta de identidad, entendiendo que son equivalentes a categorías biológicas (hombre / mujer). De esta forma, esos códigos de la masculinidad y feminidad, se transformarían en registro abierto a disposición de los cuerpos hablantes, en el contexto de los contratos consensuados temporales (PRECIADO, 2011, p.35). Esta reformulación podría presentarse como la efectiva rematerialización propuesta por Butler.

La propia reapropiación de los cuerpos del contranombre, propuesta por Preciado en el segundo principio del libro, sería una guía básica para el establecimiento de una perspectiva de rematerialización, de modo que se lograra la institución de un contrapanel con cada nuevo cuerpo, una nueva nube que había escapado a las marcas de género, con el propósito de prevenir la reevaluación de sus cuerpos como mujeres y hombres. En primer lugar, para desestabilizar el sistema heterocentrado, se elige el nombre del sexo opuesto, o nombres compuestos en masculino y femenino, funcionando estas dos fases iniciales, como forma de invalidación del sistema de reproducción heterocentrado (PRECIADO, 2011, p.35).

En la secuencia estructural histórica de la construcción de la sociedad contrasexual, el principio se guiaría por la abolición, inicialmente del contrato matrimonial y similares, como la unión estable, para perpetuar la naturalización de los roles sexuales, basándose en la lógica de que no hay contrato sexual. Podría tener el estado como testamento; en consecuencia, de los privilegios económico-sociales derivados de las condiciones masculina o femenina de los cuerpos hablantes del régimen heterocentrado; y por fin la abolición de los sistemas de transmisión y legado de los privilegios patrimoniales y económicos dotados a los cuerpos hablantes en el sistema heterocentrado (PRECIADO, 2011, p.36). Esencial la construcción de la sociedad contrasexual, según Preciado (2014, p.36), sería el principio de la universalización de prácticas estigmatizadas en el sistema heterocentrado, buscando nuevas formas de proponer sensibilidad y afecto, a través de investigaciones de alta tecnología contrasexuales.

La implementación de prácticas contrasexuales también sería importante para el sistema, sobre la base de la reevaluación del ano, como centro contrasexual universal, la difusión y distribución de prácticas subversivas de recitación de los códigos y categorías de masculinidad y feminidad naturalizadas en el ámbito heterocentrado, a partir de la resignificación y la deconstrucción de la centralidad del pene como eje de poder, a partir 
del dildo y sus variaciones sintácticas y semánticas, usados por todos los cuerpos y sujetos en los contratos (PRECIADO, 2011, p.36). La subversión del orgasmo, se incluye también en este contexto, en la medida en que visto como producción-represión heteronormativo, fragmenta el cuerpo y localiza el placer, para transformarlo en reacción natural construida ideológicamente, a través de parodias y simulaciones semejantes a los efectos asociados a él, como forma de negación de las localizaciones espacio-temporales habituales del placer, en el sentido de transformaciones generales del cuerpo (PRECIADO, 2011, p.36).

Cuando se trata de las relaciones, la sociedad contrasónica va a pautar principalmente que toda relación contrasexual será el resultado de un contrato consensuado, firmado por todos los participantes, de forma que las relaciones sin contrato serían consideradas violaciones. Los contratos serían temporales y nunca corresponderían la totalidad de la vida de los cuerpos o sujetos de hablantes, de forma que todos los cuerpos hablantes explicitaran ficciones naturalizantes, que fundamentan sus prácticas sexuales (PRECIADO, 2011, p.36). Este contrato nunca podría desembocar en relaciones de poder asimétricas y naturalizadas, debido a la característica base de que la relación contrasexual se funda en la equivalencia, requiriendo reversibilidad e intercambio de papeles.

La necesidad de separación absoluta de las actividades sexuales de las actividades de reproducción sería exigencia estructural de la sociedad, de modo que ningún contrato contrassexual conducirá la reproducción, pero ésta será libremente escogida por los cuerpos susceptibles de embarazo, o por cuerpos susceptibles de donar esperma, y ninguno de ellos los actos establecerán filiación parental natural entre los cuerpos reproductores y los cuerpos recién nacidos (PRECIADO, 2011, p.37). Los métodos anticonceptivos serán obligatorios para todos los cuerpos en edad de participar en la reproducción, siendo los sistemas de investigación y prevención de enfermedades, y la distribución gratuita de medios de prevención, los elementos esenciales para la creación y desarrollo de un sistema contrassexual (PRECIADO, 2011, p.37).

La contrasexualidad en sus principios, denuncia las actuales políticas psiquiátricas, médicas y jurídicas y los procedimientos administrativos que se refieren al cambio de sexo, denuncia la prohibición de cambio de género y nombre, y la obligación de que todo cambio de género sea acompañado de cambio de género el sexo, denunciando así el control de las prácticas transexuales por las instituciones públicas y privadas de carácter estatal heteronormativo, en la medida en que imponen cambios pautados en modelos anatómico-políticos fijos de masculinidad y feminidad, y que la contrasexualidad pretende ser tecnología de producción de cuerpos no heterocentrados (PRECIADO, 2011, p.39). Se reinventa a través de los principios de la sociedad, la comprensión del sexo y del género como cibertecnologías complejas del cuerpo (PRECIADO, 2011, 40). Consagra en la sociedad, las actividades sexuales como 
trabajo social, derecho y obligación para cualquier cuerpo, a ser practicadas regularmente por un cierto número de horas por día (PRECIADO, 2011, p. 40).

Es importante aducir que Preciado aboga por el empleo de ciertas prácticas para desmitificar las limitaciones del sexo, tratando de las prácticas de inversión contra sexual, utilizando los dildos (como complementos sexuales que auxilian el placer humano). De esa forma, Preciado busca evidenciar la utilización de los instrumentos protéticos para descubrir y aprovechar los placeres diversos, generados por la descubierta de estos mecanismos auxiliares de la sexualidad y que, las actividades sexuales, no estarían solamente en la figura del cuerpo en sí, pero en el sumar de diferentes preceptos, en algo más amplio.

La abolición de la familia nuclear como célula de producción, reproducción y consumo, debido a la relación heteronormativa en parejas estar condicionada por las finalidades reproductivas y económicas del sistema, de forma que la subversión de esa práctica se producirá a partir de prácticas contrasexuales, y de la deconstrucción de fronteras del público y privado (PRECIADO, 2011, p.41). En consecuencia, hay demanda por el principio de la construcción de pedagogía contrassexual de alta tecnología, desarrollo del saber-placer e interrupción de la historia de la humanidad como naturalización de la opresión (PRECIADO, 2011, p.42). En principio trae la consideración de que todo acto sexual sea trabajo potencial, de forma que la prostitución -solamente cuando exista contrato libre y consensual-, sea reconocida como trabajo potencial (PRECIADO, 2011, p. 42). Luego se propone repensar o procesar de una norma social y asumir, según nos induce a Butler (1993, p. 112), proporcionando así la reconstrucción completa de una sociedad de acuerdo con los principios teóricos de una sociedad contra-sexual gobernada por Paul B. Preciado.

Es válido aducir que Preciado se propone, con las prácticas sugeridas, descubrir las diferentes formas de manifestación de la sexualidad humana y de sentirse feliz por esta nueva manera de entender la sexualidad. Así como en el descubrimiento de partes del cuerpo humano antes olvidadas en la búsqueda del placer. En ese sentido, en su tarea didáctica, busca establecer un camino a ser seguido para romper con el tabú de la sexualidad reprimida y heteronormativa dominante. En el mismo modo en que afronta el modelo patriarcal, supremo e imperioso del aparato biológico masculino, es decir, la exteriorización del pene como órgano responsable de la virilidad y supervivencia de los idearios supuestamente masculinos como naturales y reproductivos.

Paul B. Preciado pretende informar a la sociedad que cada persona es detentora de un cuerpo sexual, y, por consiguiente, tiene diferentes maneras de manifestar su sexualidad y que no debemos estar juntos a preceptos restrictivos de roles atribuidos, sean por motivos biológicos, culturales, políticos, religiosos u otros. De acuerdo con Preciado, la identidad homosexual, por ejemplo: 
[...] es un accidente sistemático producido por la maquina heterosexual, y estigmatizada como antinatural, anormal y abyecta en beneficio de la estabilidad de las prácticas de producción de lo natural (PRECIADO, 2011, p. 22).

Es válido resaltar que, en el mercado del sexo heterosexual, como bien manifiesta Preciado, los hombres pueden comprar una copia de la totalidad del cuerpo femenino (las muñecas inflables), mientras que las mujeres deben contentarse con una réplica del pene. Preciado también trabaja por aclarar que el dildo es el primer indicador de la plasticidad sexual del cuerpo y de la posible modificación prostética de su contorno:

Es importante mencionar que las ideas de este libro llevan al entendimiento de las nuevas exigencias modernas de las manifestaciones sexuales no sujetas a patrones normativos excluyentes, pero sí abiertos a la diversidad de manifestaciones, desde que pactados entre sus agentes previamente (contrato), una alternativa a las relaciones cotidianas. (PRECIADO, 2011, p. 41).

Así, la obra de Preciado representa una novedosa forma de ampliar el conocimiento sobre la sexualidad:

"[...] que estudia la aparición, la formación y la utilización del dildo. Esa visión se propone localizar las tecnologías de resistencia (que por extensión llamaremos dildos y los momentos de ruptura de la cadena de producción cuerpo-placer-beneficio-cuerpo en las culturas sexuales heterosexual y queer" (PRECIADO, 2011, p. 41).

Por lo tanto, otra relevante mención dejada por el escritor ha sido, como bien explicitado más arriba, la visibilidad de la dildotecnótica.

\section{La visión queer de las Teorías del Manifiesto Contrasexual de Preciado para el cambio en el paradigma de la sexualidad humana}

La teoría queer permite pensar en la ambigüedad de la sexualidad humana, la multiplicidad y en la fluidez de las identidades sexuales y de género. Ese ideario, también, apunta para nuevas formas de pensar la cultura, el conocimiento, el poder y la educación para la inclusión participativa de inúmeras personas, que han sido excluidas en diversas sociedades a través de los siglos.

Primeramente, es imprescindible hacer alusión a la palabra de origen inglesa queer, utilizada para estigmatizar un grupo de personas asociados a vagos y maleantes, pervertidos y prostitutas, que vivían excluidos por su orientación sexual e identidad de género. Con el paso de la historia, las ideas sobre las personas queers ha propiciado a discusión social y desarrollado de nuevas definiciones para esas personas. Representando una forma diferente de conocer y de pensar sobre la diversidad sexual fuera de los modelos impositivos heteronormativos.

Ese rompimiento estructural permitió con que la teoría queer viese a tornarse una política de conocimiento cultural. Para corroborar a ese raciocinio, 
Según Jimena Furlani. Que bien definió el termino queer, destacando los estudios que tratan sobre el tema de sexualidad, hizo con que esa expresión pudiese moverse para cualquier categoría de análisis sociocultural, una vez que su esencia básica consiste en negar cualquier forma de normatividad:

Esa idea se presta tanto a las discusiones sexuales como, también, en las cuestiones raciales, étnicas, de género e etcétera. Tratase de una actitud intelectual, investigativa y crítica, de repulsa a un sistema de significación normativo (FURLANI, 2009, p. 313-4).

La teoría queer empieza a desarrollarse a finales del siglo pasado, en especial con los estudios de Judith Butler sobre género y los problemas que envuelven la cuestión y la enseñanza de técnicas sociocultural de la humanidad, en la repetición de comportamientos recibidos históricamente.

Hechos que definieron el modo de ser y de portarse de varones y mujeres, proyectando la masculinidad y feminidad de los cuerpos, rompiendo con definiciones y visibilizando todos aquellos, considerados, equivocadamente, como problemas, y que no se encuadraban en perfiles formados de género.

Las influencias bibliográficas de Preciado marcan un diverso momento para la reflexión del género, ya no solamente relacionado con la libertad reproductiva y técnicas inhibidoras del placer sexual para las mujeres, pero enfocando la libertad del placer sexual individual y al incentivo a la descubierta de nuevas técnicas para lograr satisfacer, conocer, controlar el fetiche personal y el placer de su propio cuerpo.

Como alternativas sexuales son estimuladas la masturbación y la utilización de aprovechamiento de las partes corporales como fuente de placer humano, como pueden ser las zonas del ano y la boca, que difícilmente han sido considerados como parte del sistema sexual, una vez que inviabilizan la reproducción, característica principal entendida para el sexo por generaciones pasadas.

Preciado defiende la ejemplificación de la sexualidad y los artificios utilizados con la propia evolución sexual humana, lo que antes eran considerados restrictamente la opresión y el impedimento sexual (los cinturones de castidad y afines) dan margen a las nuevas manifestaciones sexuales y a la libido sexual.

Vicisitudes que rompen con el modelo de la anatomía política repetida de la impositiva normatividad heterosexista hacía a la libertad de la expresión del placer sexual en sus más variadas ideas, como es el caso de la utilización de objetos similares, pero que de esta vez direccionados para aprovechar el sexo, el cuerpo y el auxilio del objeto introducido en la práctica, o sea la materialización como simbólica y supuesta pareja sexual. 
Oportunas también serán las ideas sobre robot y el ciborg, y sus relaciones entre el cuerpo y la máquina y viceversa respectivamente, es decir la existencia de un vínculo importante desarrollado pelas máquinas y el cuerpo humano y su sexualidad, como una prótesis inteligente usada para lograr la orgásmica concretización del placer.

En este momento reiteramos que la obra de Preciado está contextualizada dentro del feminismo moderno, también denominada como nuevo movimiento, o la tercera ola del feminismo. Período este que comienza, según la opinión de Garaizabal: “[...] en los años sesenta en España, y se desarrolla a partir de 1975, con la muerte del General Francisco Franco" (GARAIZABAL, 2009).

Es importante afirmar que el nuevo feminismo supuso un cambio fundamental en la comprensión del género como construcción social, cultural, político, religioso, histórico, etcétera. En ese sentido, el género pasa a ser comprendido como un producto multidisciplinar, con diversas variantes que inciden sobre él, y que contribuyen, o no, para la inclusión de principios de igualdad de trato, acceso a derechos, implementando la justicia y democracia.

Ese momento único marca el feminismo moderno como movimiento cuestionador de los roles históricos establecidos de generación a generación, que hicieron de la definición de naturalidad de los géneros, y de los sexos, una visión ultrapasada. Por lo tanto, creando la necesidad de combatir la idea de natural, cuestionándola y formando la interpretación de que los géneros recibían una fuerte influencia de valores históricos, religiosos, cultural que impedían, sobretodo, su verdadero y amplio entendimiento.

Es válido relatar que las relaciones de género están intrínsecamente relacionadas con la manera de portarse de las personas en la vida en sociedad, siendo por lo tanto influenciados por construido socio cultural. En realidad, están en las acciones que realizamos y, también, en nuestras relaciones con las demás personas.

El sistema de sexo-género es un sistema de escritura. El cuerpo es un texto socialmente construido, un archivo orgánico de la historia de la humanidad como historia de la producción-reproducción sexual, en la que ciertos códigos se naturalizan, otros quedan elípticos y otros son sistemáticamente eliminados y tachados (PRECIADO, 2011, p.18).

En la gran mayoría de las sociedades, se entiende que las mujeres son sujetos de la historia $\mathrm{y}$, por eso, hacen parte indispensable para la consolidación de una sociedad más correcta e igualitaria. La obligación de respetar los derechos y los deberes, es decir, de tener ciudadanía constituye un importante papel que pertenece a cada individuo en sociedad, donde varones y mujeres son iguales en dignidad y merecen lo mismo trato y respeto.

Por ese motivo, enfocamos la necesidad de trabajar la concientización general de estas sociedades, donde los varones, detentores de una forma de dominación más amplia, puedan auxiliar que las mujeres tengan su efectiva participación en los más diversos sectores 
(económica, política, social), simplemente en igualdad de condiciones. Esto es lo que enfatiza el ideal feminista, donde no existe la superioridad de la figura de la mujer en relación al varón y sí, la igual participación entre ambos.

La actualidad hace con que las cuestiones de género sean comprendidas como un proceso mucho más amplio que se adecua a las nuevas exigencias democráticas, previstas y efectivas en diversas Constituciones por el mundo. Las nuevas realidades emplean la integración del género y la participación igualitaria entre las personas, evidenciando la participación ciudadana y la reivindicación social por mejores condiciones de vida para todos que viven excluidos.

\section{Un recorrido por la obra de Beatriz Preciado y su relación con la sexualidad humana. La necesidad de romperse los estigmas del sexo: la expresión del feminismo moderno}

La sexualidad está relacionada con elaboraciones culturales sobre el placer y los intercambios sociales y corporales que comprenden desde el erotismo, el deseo, hasta nociones relativas a la salud, la reproducción, al uso de tecnologías y el ejercicio del poder en sociedad. Las definiciones actuales de la sexualidad abarcan, en las ciencias sociales, significados, ideas, deseos, sensaciones, emociones, experiencias, conductas, prohibiciones, modelos y fantasías que son configurados de modos diversos en distintos contextos sociales y periodos históricos. Es un concepto dinámico que va evolucionando y que está sujeto a diversos usos, múltiples y contradictorias interpretaciones y que se encuentra sujeto a debates y a disputas políticas (MANUAL DE COMUNICAÇÃO LGBT, 2010, p. 9).

Paul B. Preciado defiende la idea de que la sexualidad es fruto de la construcción del comportamiento humano sociopolítico, influenciado por el tiempo histórico. Es un constructo amplio, de diferentes manifestaciones y no solamente está en la división restricta del masculino o femenino. Es algo que está más allá de estos dos términos, existe sí la necesidad de considerar una serie de factores que influencian en la manifestación sexual del cuerpo humano, sea por motivos biológicos (genéticos o cromosómicos) o por otras variantes.

Al igual que Preciado, Patricia Hill Collins en Black Sexual Politics (2004) nos llama a comprender varios factores que influyen en la manifestación sexual humana, "Hablar sobre género no significa centrarse únicamente en los problemas de las mujeres. Las experiencias de los hombres también están profundamente relacionadas con el género. Así, la ideología de género no solo crea ideas sobre la feminidad sino que también da forma a las concepciones de la masculinidad" (COLLINS, 2004, p. 6). Por lo tanto, la necesidad de entender la sexualidad no como una función biológica es evidente; pero como un sistema de ideas y prácticas sociales profundamente involucradas en la formación de desigualdades sociales (COLLINS, 2004, p. 6). Collins (2004) comprende una gama de agentes sociopolíticos, que interactúan en la construcción e identidad de género, categorías que no pueden analizarse sin una comprensión efectiva de la política sexual, definieron "un conjunto de ideas y prácticas sociales moldeadas 
por género, raza y la sexualidad que enmarca el tratamiento de todos los hombres y mujeres, así como la forma en que los hombres y las mujeres son percibidos y tratados por otros" (COLLINS, 2004, p. 6).

Partiendo de la comprensión de la construcción de una ideología de género negra como justificación de los patrones de discriminación racial, Collins (2004, p.6) amplía el debate de género al explicar cómo para los afroamericanos se intensifica la relación entre género y raza, produciendo una ideología de género negro que da forma a ideas sobre masculinidad y feminidad negras. Esta política sexual, según Collins, “ocurrirá en la intersección particular de género, raza y sexualidad que enfrentan los afroamericanos" (COLLINS, 2004, p. 7), no solo se limita a los afroamericanos, sino que se expande para abarcar "Una amplia constelación de grupos sociales, como mujeres blancas, hombres latinos, inmigrantes asiáticos homosexuales y lesbianas, estadounidenses ricos, ancianos indígenas y madres asiáticas jóvenes casadas" (COLLINS, 2004, p. 7), que encuentran políticas sexuales distintas con base a sus sistemas de género, raza y sexualidad.

Es debido a estas contribuciones recientes que existe la necesidad de comprender los paquetes de sexualidad en multiplicidad desde varias áreas, prestando atención a las intersecciones que impregnan el debate, estimulando una visión más explícita de los problemas sexuales. Es una construcción que se refiere a los hábitos, prácticas, comportamientos, pensamientos de una persona con el tema del sexo y su posterior relación social. En esta búsqueda, varios otros autores intentan definir y estudiar la sexualidad humana, la biología, la medicina, la teología, el psicoanálisis, la sociología, la antropología, el derecho y muchos otros.

Como bien aporta Félix Sánchez, los seres humanos, en efecto son:

1) individuos diferentes genética y culturalmente, 2) tienen una identidad sexual que depende de las características biológicas del hombre y de la mujer, 3) tienen una identidad sexual sobre las que las otras culturas han construyendo unos roles de género, case siempre discriminatorios para con las mujeres, que regulan numerosos aspectos de la vida social de las personas, 4) tienen, como ocurre en otras especies sexuadas, una fuerte motivación sexual (deseo y atracción) e su actividad sexual está premiada (placer sexual), 5) tienen la característica de que la sexualidad es una dimensión más rica y compleja que nos limita a una mera función reproductora (SÁNCHEZ, 2005, p. 20-2).

De este modo, los seres humanos son especiales por detener el conocimiento inherente a la razón que les permite distinguir de otros animales por su raciocinio e entendimiento sobre las cosas y el conocimiento de su propia existencia como humano. Sumados a esas características, las personas tienen la posibilidad de comprender el acto sexual/emocional no solamente como expresión de la reproducción humana, pero como camino hacia la felicidad personal u colectiva, en respeto a la dignidad humana y al pleno desarrollo del placer físico/ emocional. 


\section{Consideraciones finales}

Este estudio se ha preguntado cómo el trabajo de Manifestación de contraste ha contribuido a la comprensión del género y la sexualidad. Hemos entendido que los roles se construyen en la sociedad. Para los cambios en los paradigmas de las desigualdades entre hombres y mujeres, ha habido muchos reclamos de movimientos feministas en los últimos años.

Vale la pena señalar que estos esfuerzos han proporcionado una visibilidad globalizada de la cuestión de género y sus implicaciones en la sexualidad de las mujeres en un contexto más amplio y liberador. A partir de este momento, el sexo supera el rango restringido de reproducción, aumentando el descubrimiento de la sexualidad, el placer del sexo y la introducción de alternativas a las prácticas sexuales permitidas por el imperio heteronormativo.

Estas diversas teorías no se resumen en este momento en las reflexiones iniciadas en el texto, pero las preguntas que surgen de esta interrelación están latentes. ¿Cuáles serán las agendas interseccionales entre la manifestación de countersex y otras teorías feministas? ¿Cuánto entienden los principios establecidos por Preciado las múltiples sexualidades que existen? ¿Qué contribución tiene una que está ausente de reflexión? ¿Hay alguna reflexión que no impulse cambios paradigmáticos? Como toda investigación, las preguntas son más amplias que las conclusiones y nos llevan a un mayor análisis y debate.

Es por el movimiento feminista en la historia de las mujeres que, junto con otras, grupos de víctimas de exclusión participativa, destacando una situación problemática que enfrentan hombres y mujeres: la desaparición femenina y la privación social de las mujeres en sociedades predominantemente. Acceso patriarcal y participativo limitado a estas personas.

El estudio incluye ciertos objetivos para la consolidación de mecanismos para la integración socio-sexual de las mujeres en sus diversas manifestaciones, a través de orientaciones sexuales (heterosexualidad, bisexualidad, transexuales, homosexualidad, etc.) o mediante identidades de género.

De esta manera, si se ha demostrado que la inclusión de grupos excluidos en la sociedad está vinculada a varios factores cruciales: será: la necesidad de intercambio en la ilustración de la subordinación que está sujeta a los grupos de mujeres, así como el tema temático a la misma La deconstrucción de las políticas sexuales y otras políticas que se cruzan con el género, la raza y la clase. Homosexualidad y sexualidad de las personas.

Del mismo modo, requeriría la adopción de disposiciones legales normativas y la creación de políticas públicas y privadas, así como el desarrollo de nuevos estudios que hayan promovido el empoderamiento de las mujeres, para evitar que las mujeres estén indefensas en sus formas y demandas, en particular en relación a la sexualidad que los rodea. 
Todas estas ideas son válidas para respetar y construir una sociedad más justa y equitativa en cuestiones de género e identidad, orientación sexual y educación para la diversidad sexual humana. Estas pautas, así como el trabajo de Preciado, sirven de inspiración para la reflexión y el intercambio en diversas sociedades que comparten dificultades extremas para lograr la igualdad real de trato entre géneros, con acceso a los mismos obstáculos y obstáculos discriminatorios.

Para lograr estos objetivos, será necesario cambiar las actitudes presentes en la sexualidad humana, actitudes que son evidentes, por ejemplo: la jerarquía de los sexos (hombres supuestamente superiores a las mujeres), la eliminación de los obstáculos discriminatorios presentes en los preceptos legales. El comportamiento histórico social (cultura, religión, política, raza, etc.).

Es imperativo agregar que las diferentes formas de orientación humana, ya sean homosexuales, heterosexuales y bisexuales, no son el resultado de una decisión personal, pero algunas personas la llenan erróneamente.

Para todos aquellos que no los entienden, las orientaciones sexuales simplemente se manifiestan, y depende de ellos aceptarlas o negarlas. Por lo tanto, corresponde a la sociedad organizada y a los estados permitir que estas personas sean libres de ver sus sexualidades, obstáculos, acceso a ellas, pero nada menos.

\section{Referencias}

BUTLER, Judith. Bodies that matter. On the discoursive limits of "sex". New York, USA: Routledge, 1993.

CARRILLO, Jesús. "Entrevista com Beatriz Preciado". REVISTA POIÉSIS, Niterói, n. 15, julho, p. 47-71, 2010.

COLLINS, Patricia Hill. Black Sexual Politics: African American, Gender and The New Racism. New York, USA: Routledge, 2004.

FURLANI, Jimena. "Direitos Humanos, Direitos Sexuais e Pedagogia Queer: o que essas abordagens têm a dizer à educação sexual? ” In: JUNQUEIRA, Rogério Diniz (Org.). Diversidade Sexual na Educação: problematizações sobre a homofobia nas escolas. Brasília. Brasil: Coleção Educação para todos, MEC/UNESCO, p. 293-324, 2009.

GARAIZABAL, Cristina. "Debates feministas sobre la sexualidade”. Disponible en: http:// Www.aldarte.org/comun/imagenes/documentos/Debates_feministas_sobre_la_sexualidad-_ Cristina_Garaizabal.pdf, acceso en: 16 abr. 2019.

GIL, Antônio Carlos. Métodos e técnicas de pesquisa social. 6. ed. São Paulo: Atlas, 2008. 
MAQUIEIRA, Virginia D`Angelo. Democracia, feminismo y Universidad en el siglo XXI. Madrid, España: Instituto Universitario de Estudios de la Mujer, 2005.

MANUAL DE COMUNICAÇÃO LGBT. Lésbicas, Gays, Bissexuais, Travestis e Transexuais. Brasilia, Brasil: ABGLT, 2010.

MARCONI, Marina de Andrade; LAKATOS, Eva Maria. Fundamentos de metodologia cientifica. 7. ed. São Paulo: Atlas, 2010.

PÉREZ, Moira. "La cadena sexo-género-revolución". Revista Estudos Feministas. Florianópolis, Brasil, 25(2)562, p. 435-451, maio/agos. 2017.

PRECIADO, Beatriz. Manifiesto contrasexual. Barcelona, España: Anagrama, Colección Argumentos, 2011.

SÁNCHEZ, Félix López. La educación sexual. Madrid, España: Biblioteca Nueva, 2005.

SEGARRA, Marta; CARABÍ, Ángels. Nuevas masculinidades. Construyendo nuevas masculinidades: una introducción. Barcelona, España: Icaria, mujeres y culturas, 2000.

SILVA, Joseli Maria; ORNAT, Marcio Jose. "Geografias Lésbicas: uma Entrevista com Eduarda Ferreira". Revista Latino Americana de Geografia e Gênero, Ponta Grossa, v. 8, n. 1., p. 424-434, 2017.

VERBICARO SOARES, Douglas. La libertad sexual en la sociedad: especial referencia a la homosexualidad en las Fuerzas Armadas Brasileñas. Tesis Doctoral del Programa de Doctorado Pasado y Presente de los Derechos Humanos, Universidad de Salamanca USAL, Salamanca, España, 2015.

YOUTUBE. Beatriz Preciado en Redes despachando su crítica al biopoder. Disponible en: http://www.youtube.com/watch? $\mathrm{v}=\mathrm{W} 8 \mathrm{wfc} 2 \mathrm{JNMd}$, acceso en: 9 maio 2019. 